04

\title{
Начальные стадии формирования импульсного разряда в промежутке с геометрией острие-плоскость в предварительно ионизированном аргоне
}

\author{
(C) В.С. Курбанисмаилов ${ }^{1}$, Д.В. Терешонок ${ }^{2}$, Г.Б. Рагимханов ${ }^{1}$, 3.Р. Халикова ${ }^{1}$ \\ ${ }^{1}$ Дагестанский государственный университет, Махачкала, Россия \\ ${ }^{2}$ Объединенный институт высоких температур РАН, Москва, Россия \\ E-mail: gb-r@mail.ru
}

Поступило в Редакцию 28 октября 2021 г.

В окончательной редакции 14 декабря 2021 г.

Принято к публикации 16 декабря 2021г.

На основе двумерной осесимметричной диффузионно-дрейфовой модели выполнено исследование влияния начальных условий на особенности формирования и развития анодонаправленной волны ионизации между двумя электродами с геометрией промежутка острие-плоскость в аргоне при атмосферном давлении.

Ключевые слова: газовый разряд, низкотемпературная плазма, пробой газов, волны ионизации, моделирование.

DOI: 10.21883/PJTF.2022.05.52157.19067

Несмотря на большое число работ, посвященных исследованию импульсных объемных разрядов, многие вопросы, связанные с физикой формирования начальных стадий, вызывают научные дискуссии [1-4], в том числе и для инертных газов $[5,6]$.

В настоящей работе в условиях равномерной предварительной ионизации аргона при атмосферном давлении выполнено расчетно-теоретическое исследование начальной стадии формирования импульсного разряда в аргоне в межэлектродном промежутке с геометрией острие-плоскость. Острие (катод) представляло собой прямой цилиндр радиусом $1 \mathrm{~mm}$ и длиной $1 \mathrm{~cm}$, расстояние от острия до плоскости было равно $8.5 \mathrm{~mm}$.

Такая постановка задачи, по мнению авторов, представляет интерес ввиду геометрии катода. Как правило, при моделировании катод задают в виде иглы с заостренным или закругленным кончиком, что автоматически дает максимальное значение напряженности поля на оси разрядного промежутка. В настоящей работе использование прямого цилиндра создает предпосылки к торообразной геометрии ионизационной волны, так как максимальная напряженность поля находится вблизи торца цилиндра.

Моделирование выполнено в двумерной осесимметричной постановке с начальной концентрацией электронов и атомарных ионов $10^{8} \mathrm{~cm}^{-3}$ во всей расчетной области. Напряжение на электродах в течение всего времени расчета составляло $5 \mathrm{kV}$.

Прямоугольная расчетная сетка по радиусу была со сгущением к оси разряда с числом ячеек $N_{r}=1050$. В межэлектродном промежутке сетка сгущалась вблизи электродов $N_{z}=1900$.

Газоразрядная плазма рассматривается как сплошная многокомпонентная среда, состоящая из нейтральных атомов $(\mathrm{Ar})$, электронов $(e)$, возбужденных атомов $\left(\mathrm{Ar}^{*}\right)$ с энергией возбуждения $11.5 \mathrm{eV}$, атомарных $\left(\mathrm{Ar}^{+}\right)$и молекулярных $\left(\mathrm{Ar}_{2}^{+}\right)$ионов. Кинетика рассматриваемых процессов, константы соответствующих реакций (за исключением прямой ионизации и возбуждения) были взяты из работы [7].

Приведенная далее система уравнений включает в себя уравнения баланса заряженных и возбужденных частиц, уравнение для энергии электронов и уравнение Пуассона [7-10]. Нагрев нейтрального газа не учитывался. Температура тяжелых частиц в процессе счета предполагалась равной температуре нейтрального газа $(300 \mathrm{~K})$.

$$
\begin{gathered}
\frac{\partial n}{\partial t}+\nabla \cdot \boldsymbol{\Gamma}=S, \\
\boldsymbol{\Gamma}=q n \mu \mathbf{E}-D \nabla n, \\
\frac{\partial}{\partial t}\left(\frac{3}{2} n_{e} k_{\mathrm{B}} T_{e}\right)+\nabla \cdot \mathbf{F}=\mathbf{j}_{e} \cdot \mathbf{E}+S_{e}, \\
\mathbf{F}=\frac{5}{2} k_{\mathrm{B}} T_{e} \boldsymbol{\Gamma}_{e}-\lambda_{e} \nabla T_{e}, \\
\lambda_{e}=\frac{5}{2} n_{e} D_{e}, \\
\nabla \cdot \mathbf{E}=\frac{e\left(n_{\mathrm{Ar}^{+}}+n_{\mathrm{Ar}_{2}^{+}}-n_{e}\right)}{\varepsilon_{0}},
\end{gathered}
$$

где $n, \boldsymbol{\Gamma}, \mu, D-$ концентрация, поток, подвижность и коэффициент диффузии соответствующих компонентов плазмы, $e-$ заряд электрона, $k_{\mathrm{B}}-$ постоянная Больцмана, $T_{e}$ - температура электронов, $\lambda_{e}, D_{e}-$ теплопроводность и коэффициент диффузии электронов, которые определялись приведенным локальным полем $E / N$ из BOLSIG $+[11], n_{e}, n_{\mathrm{Ar}^{+}}, n_{\mathrm{Ar}_{2}^{+}}-$концентрации электронов, атомарных и молекулярных ионов, $S-$ источник рождения и гибели рассматриваемых частиц в плазме, $S_{e}-$ упругие и неупругие потери электронов, 

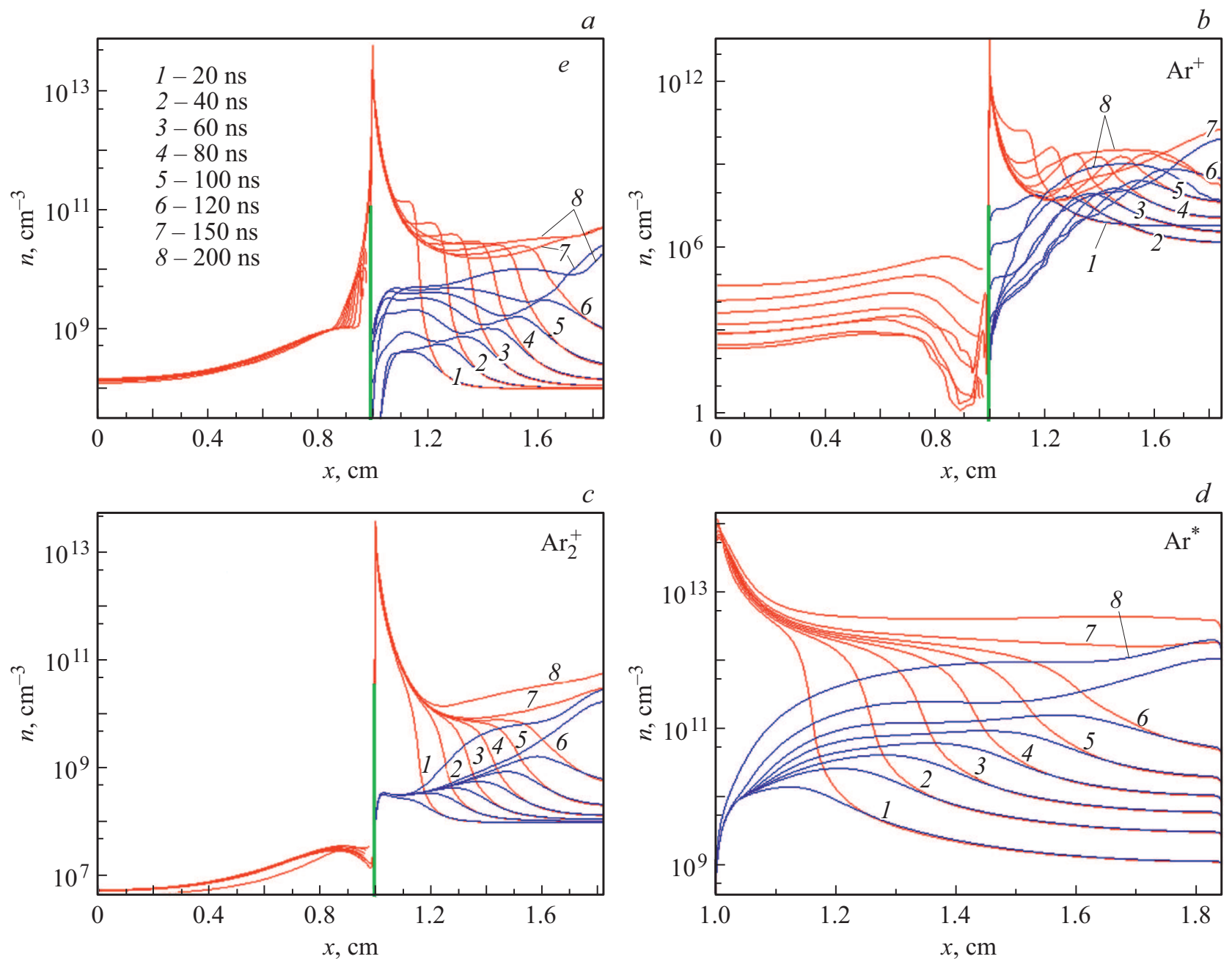

Рис. 1. Распределение концентрации электронов $(a)$, атомарных ионов аргона $\mathrm{Ar}^{+}(b)$, молекулярных ионов аргона $\mathrm{Ar}_{2}^{+}(c)$, возбужденных атомов аргона $\operatorname{Ar}^{*}(d)$ в разрядном промежутке для различных моментов времени. Синие линии - $r=0$, красные $-r=0.1 \mathrm{~cm}$. Цветной вариант рисунка представлен в электронной версии статьи.

$\mathbf{j}_{e}-$ плотность тока электронов, $\mathbf{E}-$ напряженность электрического поля. Для ионов $q=+1$, для электронов $q=-1$, для возбужденных частиц $q=0$.

Константы возбуждения и прямой ионизации определялись приведенным локальным полем $E / N$ из BOLSIG+ [11]. Остальные константы задавались как функции $T_{e}$.

Коэффициенты подвижности для ионов и коэффициент диффузии возбужденных частиц в собственном газе были взяты из [12].

Граничные условия на катоде для потенциала, заряженных и возбужденных частиц $n^{*}$ (индекс $i$ относится к атомарным и молекулярным ионам)

$$
\begin{gathered}
\varphi_{c}=0, \quad \frac{\partial n_{i}}{\partial z}=0, \quad \boldsymbol{\Gamma}_{e}=-\gamma \sum_{i} \boldsymbol{\Gamma}_{i}, \\
n^{*}=0, \quad \frac{3}{2} k_{\mathrm{B}} T_{e}=I-2 \varphi_{W},
\end{gathered}
$$

на аноде

$$
\varphi_{a}=V_{a}, \frac{\partial n_{e}}{\partial z}=0, \frac{\partial T_{e}}{\partial z}=0, n_{i}=0, n^{*}=0,
$$

на боковых гранях расчетной области

$$
\frac{\partial \varphi}{\partial r}=0, \frac{\partial n_{e}}{\partial r}=0, \frac{\partial n_{i}}{\partial r}=0, \frac{\partial n^{*}}{\partial r}=0, \frac{\partial T_{e}}{\partial r}=0,
$$

где $\gamma=0.1-$ второй коэффициент Таунсенда, $I=15.76 \mathrm{eV}-$ потенциал ионизации аргона, $\varphi_{W}=4.5 \mathrm{eV}$ - работа выхода катода, $V_{a}-$ потенциал анода. Для ион-электронной эмиссии учитывался поток на катод как атомарных, так и молекулярных ионов.

Решение конвективно-диффузионных уравнений получалось методом конечных объемов [13]. Уравнение Пуассона решалось итерационным методом переменных направлений.

На рис. 1 приведены характерные распределения концентрации электронов $(a)$, атомарных ионов аргона 


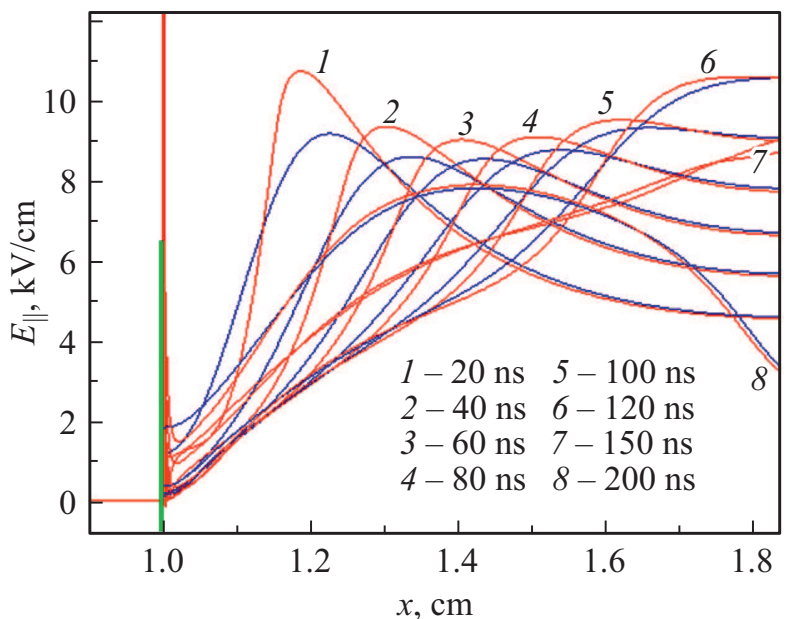

Рис. 2. Распределение амплитуды продольной составляющей напряженности электрического поля $E$ в разрядном промежутке для различных моментов времени. Синие линии $-r=0$, красные $-r=0.1 \mathrm{~cm}$. Цветной вариант рисунка представлен в электронной версии статьи.

$\mathrm{Ar}^{+}(b)$, молекулярных ионов аргона $\mathrm{Ar}_{2}^{+}(c)$, а также возбужденных атомов аргона $\operatorname{Ar}^{*}(d)$ в промежутке для различных моментов времени в случаях $r=0$ и $0.1 \mathrm{~cm}$.

Как показывает анализ результатов расчета (рис. $1, a-d)$, на начальном этапе формирования разряда в разрядном промежутке образуется анодонаправленная волна ионизации. Следует отметить, что концентрации заряженных и возбужденных частиц в промежутке имеют максимальные значения вблизи края острия на расстоянии $0.1 \mathrm{~cm}$ от оси разряда, что объясняется краевыми эффектами, поскольку острие имеет форму цилиндра, а следовательно, поле на краю острия выше (рис. 2), чем на оси, что ведет к более интенсивной ионизации.

С течением времени концентрация атомарных ионов аргона $\mathrm{Ar}^{+}$(рис. $\left.1, b\right)$ на оси $(r=0 \mathrm{~cm})$ в промежутке возрастает и максимум смешается в сторону анода. К моменту времени $150 \mathrm{~ns}$ концентрация максимальна вблизи анода, а к $200 \mathrm{~ns}$ концентрация ионов максимальна в середине промежутка между острием и анодом. Аналогичная картина прослеживается для концентрации молекулярных ионов $\mathrm{Ar}_{2}^{+}$(рис. 1,c), но концентрация $\mathrm{Ar}_{2}^{+}$в промежутке выше, чем концентрации $\mathrm{Ar}^{+}$и возбужденных атомов аргона $\mathrm{Ar}^{*}$ (рис. $\left.1, d\right)$, что указывает на высокую интенсивность процессов образования молекулярных ионов в промежутке в процессе конверсии. При высоких давлениях и низкой температуре газа (на начальной стадии формирования газ не успевает нагреться) идет наработка молекулярных ионов.

Как видно из полученных результатов, для значения прикладываемого к промежутку напряжения $V_{a}=5 \mathrm{kV}$, несмотря на сильную неоднородность электрического поля $E$ (рис. 2) за счет характерной геометрии разрядного промежутка, скорость анодонаправленной волны в интервале времени от 20 до $120 \mathrm{~ns}$ (рис. 2) остается постоянной и близка к значению $5 \cdot 10^{6} \mathrm{~cm} / \mathrm{s}$. В момент прихода волны на анод концентрация электронов на оси разряда максимальна вблизи анода, однако на краю острия (на расстоянии $0.1 \mathrm{~cm}$ от центра) концентрация электронов остается максимальной в промежутке, что объясняется высоким значением $E$ (рис. 2) по сравнению с полем на оси разряда.

Можно отметить качественное совпадение с другими расчетами поведения напряженности электрического поля $E$ вдоль разряда для аргона в геометрии острие-плоскость [5], где сначала $E$ падает, а затем растет по мере приближения к аноду.

В работе выполнено двумерное моделирование формирования импульсного разряда в аргоне атмосферного давления в промежутке с геометрией острие-плоскость.

Показано, что на стадии формирования в результате реакции $\mathrm{Ar}+\mathrm{Ar}+\mathrm{Ar}^{+} \rightarrow \mathrm{Ar}+\mathrm{Ar}_{2}^{+}$концентрация молекулярных ионов аргона $\mathrm{Ar}_{2}^{+}$существенно превосходит концентрацию атомарных ионов $\mathrm{Ar}^{+}$, что обусловлено слабым разрушением $\mathrm{Ar}_{2}^{+}$ввиду отсутствия нагрева газа. Установлено, что в разрядном промежутке на временах до $200 \mathrm{~ns}$ формируется анодонаправленная волна ионизации. При этом, несмотря на сильную неоднородность электрического поля, скорость анодонаправленной волны остается постоянной и равной $5 \cdot 10^{6} \mathrm{~cm} / \mathrm{s}$.

\section{Финансирование работы}

Работа выполнена при финансовой поддержке Российского фонда фундаментальных исследований (грант № 19-08-00333a).

\section{Конфликт интересов}

Авторы заявляют, что у них нет конфликта интересов.

\section{Список литературы}

[1] Ю.Д. Королев, Г.А. Месяц, Физика импульсного пробоя газов (Наука, М., 1991).

[2] В.Ф. Тарасенко, Е.Х. Бакшт, А.Г. Бураченко, М.И. Ломаев, Д.А. Сорокин, Ю.В. Шутько, Письма в ЖТФ, 36 (8), 60 (2010). [V.F. Tarasenko, E. Baksht, A.G. Burachenko, M.I. Lomaev, D.A. Sorokin, Yu.V. Shut'ko, Tech. Phys. Lett., 36 (4), 375 (2010). DOI: 10.1134/S1063785010040255].

[3] G.V. Naidis, V.F. Tarasenko, N.Yu. Babaeva, M.I. Lomaev, Plasma Sources Sci. Technol., 27 (1), 013001 (2018). DOI: $10.1088 / 1361-6595 / \mathrm{aaa} 072$

[4] В.В. Осипов, УФН, 170 (3), 225 (2000). DOI: $10.3367 /$ UFNr.0170.200003a.0225 [V.V. Osipov, Phys. Usp., 43 (3), 221 (2000). DOI: 10.1070/pu2000v043n03ABEH000602].

[5] Y. Sato, K. Ishikawa, T. Tsutsumi, A. Ui, M. Akita, S. Oka, M. Hori, J. Phys. D: Appl. Phys., 53 (26), 265204 (2020). DOI: $10.1088 / 1361-6463 / a b 7 d f 0$ 
[6] A. Sobota, F. Manders, E.M. van Veldhuizen, Jan van Dijk, M. Haverlag, IEEE Trans. Plasma Sci., 38 (9), 2289 (2010). DOI: 10.1109/TPS.2010.2056934

[7] M. Baeva, A. Bösel, J. Ehlbeck, D. Loffhagen, Phys. Rev. E, 85 (5), 056404 (2012). DOI: 10.1103/PhysRevE.85.056404

[8] Д.В. Терешонок, Письма в ЖТФ, 40 (3), 83 (2014). [D.V. Tereshonok, Tech. Phys. Lett., 40 (2), 135 (2014). DOI: $10.1134 / \mathrm{S} 106378501402014 \mathrm{X}$.

[9] С.Т. Суржиков, Физическая механика газовых разрядов (Изд-во МГТУ им. Н.Э. Баумана, М., 2006).

[10] В.С. Курбанисмаилов, О.А. Омаров, Г.Б. Рагимханов, Д.В. Терешонок, Письма в ЖТФ, 43 (18), 73 (2017). DOI: $10.21883 /$ PJTF.2017.18.45036.16844

[V.S. Kurbanismailov, O.A. Omarov, G.B. Ragimkhanov, D.V. Tereshonok, Tech. Phys. Lett., 43 (9), 853 (2017). DOI: $10.1134 / \mathrm{S} 1063785017090206]$.

[11] G.J.M. Hagelaar, L.C. Pitchford, Plasma Sources Sci. Technol., 14 (4), 722 (2005). DOI: 10.1088/0963-0252/14/4/011

[12] Б.М. Смирнов, Свойства газоразрядной плазмы (Изд-во Политехн. ун-та, СПб., 2010).

[13] J. Teunissen, Plasma Sources Sci. Technol., 29 (1), 015010 (2020). DOI: 10.1088/1361-6595/ab6757 